\title{
Spectrum handoff analysis for multiple secondary users in cognitive radio networks
}

\author{
Ban Bakhtyar Mahmud Shawkat, Asaad M. Jassim Al-Hindawi, Akram Hatam Shadir \\ Department of Communication Engineering, Sulaimani Polytechnic University, Iraq
}

\begin{tabular}{l}
\hline \hline Article Info \\
\hline Article history: \\
Received Jan 26, 2020 \\
Revised Mar 15, 2020 \\
Accepted Apr 5, 2020 \\
\hline
\end{tabular}

\section{Keywords:}

Cognitive radio network

Queuing model

Spectrum handoff in CRN

\begin{abstract}
The cognitive radio network permits secondary users to reach unused spectrum of primary users. In this paper, a mixed preemptive/non-preemptive resume priority M/G/1 queuing model is proposed for characterizing multiple handoff delay and to minimize the extended data delivery time of secondary users that has experienced multiple spectrum handoff during its transmission. The proposed queuing model supports delay-sensitive secondary user applications. The secondary users' traffic is classified into two priority classes, the highest priority class for delay sensitive services and the lowest priority class for delay insensitive services. Furthermore, the proposed model assigns higher priority for the interrupted secondary users over uninterrupted secondary users for each class of secondary users in order to minimize the handoff delays for secondary users that experience multiple interruptions. Analytical formulas for the average extended data delivery time are derived for two different proactive spectrum handoff strategies (always stay and always change strategies) for both classes of secondary users. Simulation of proposed system model was performed to validate the analytical results and a good agreement was obtained. The performance of the suggested model is assessed and compared with other spectrum handoff models. Numerical results illustrate that the proposed system model outperforms existing models and can reduce the extended data delivery time for the secondary users.
\end{abstract}

Copyright $\odot 2020$ Institute of Advanced Engineering and Science. All rights reserved.

\section{Corresponding Author:}

Ban Bakhtyar Mahmud Shawkat, Department of Communication Engineering,

Sulaimani Polytechnic University, Sulaimani, Kurdistan region, Iraq. Email: ban.bakhtyar@spu.edu.iq

\section{INTRODUCTION}

Radio spectrum needs to be managed as it is a finite natural resource. To support different wireless applications and prevent them from interfering with each other, spectrum regulators have followed fixed spectrum access policy (FSA) [1]. With FSA, each part of the spectrum with specific bandwidth is assigned to a licensed user or several licensed users. However, the licensed spectrum is underused [2, 3].

To increase spectrum usage, dynamic spectrum access (DSA) has been suggested as an alternative policy to utilize the spectrum holes and reduce the problem of spectrum lack. The secondary users (SUs) search for unoccupied spectrum bands dynamically and access them temporarily for wireless communications. SUs must vacate the spectrum band whenever primary users (PUs) start using the band [4]. The technology of cognitive radio networks (CRNs) is the key enabling technology of DSA [5].

Mitola [6], was the first who introduced the term cognitive radio which is a new innovation that permits SUs to utilize PUs unused licensed spectrum bands to increase the utilization of the scarce frequency spectrum. During the transmission period PUs have the right to pre-empt the SUs when PUs arrive at their channel, SUs must continue its transmission at another vacant channel, this process in CRNs is called 
spectrum handoff $[7,8]$, that is providing reliable transmissions for SUs that have been interrupted by PUs, and helping them to continue their uncompleted transmission which can minimize the performance degradation during spectrum handoff by providing smooth and fast switching [9].

Spectrum handoff schemes in CRN can be classified into switching and non-switching handoff. Figure 1 shows spectrum handoff schemes classification. In non-switching handoff scheme, when a PU arrives at its target channel the SU pauses its transmission and waits on the same channel until all PUs finish their transmission and the channel becomes idle again then the SU resumes its undone transmission [10]. Because the SU does not change its channel, there is no delay for channel switching therefore the handoff delay in the non-switching technique is only the time which the secondary user spends waiting on the channel.

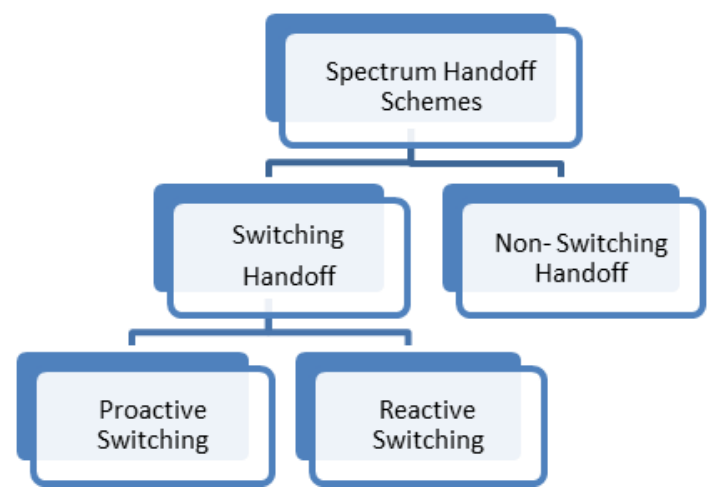

Figure 1. Spectrum handoff schemes

For the switching handoff technique, it can further be classified into proactive switching and reactive switching. Based on the decision time for selecting the desired channels [8]. In spectrum handoff of proactive-decision, secondary users prepare desired channels before the beginning of their sending information by regularly monitoring all channels for the purpose of collecting channel information. Then, SU decides which channel is the best for the next spectrum handoffs according to results obtained from long observation period. While in reactive spectrum handoff, SU searches for target channel after the interruption, after this search the SU will continue the interrupted transmission on one of the target channels.

In the literature, most of the existing works have used $\mathrm{M} / \mathrm{G} / 1$ priority queueing model to model spectrum handoff. In [11-14] the authors have considered one queue for primary users and one queue for secondary users. All types of SUs waits in the same queue and have the same priority. However, the same priority is given to all SUs in the unitary model. This makes the queueing model inappropriate for the networks that are having delay-sensitive applications. The different wireless communication services, have different delay requirements. If all SUs types have the same priority to access the channels without differentiation, then the performance of some delay-sensitive services might not be achieved while some delay-insensitive services might be enhanced indifferently.

In [15-18] the authors divide the SUs to multiple classes according to their priority. The queue of highest priority is for PUs and there is N number of queues for N Classes of SUs. In [17, 18] PUs have the highest priority and can pre-empts SUs, SUs that are having higher priorities can pre-empt the transmissions of SUs with lower priorities. However, this queueing model might cause deterioration in the total transmission delay over CRNs because of overly frequent spectrum handoff while in $[15,16]$ the SUs cannot interrupt the ongoing transmission of other SUs of lower classes so as to prevent multiple interruption for lower priority SUs. Although the multiclass model can solve the problem of delay sensitive networks by assigning higher priority to the SUs with delay sensitive service over SUs with delay insensitive services but, both the unitary model and the multiclass model that have been mentioned do not assign priority to interrupted users over uninterrupted users. Multiple spectrum handoffs will severely degrade the interrupted users ' QoS because it increases the handoff delay and overall service time. Assigning higher priority to the interrupted users over newly arriving uninterrupted users can minimize the average service time and show significant performance gains.

In $[9,19]$ there are three priority classes. The highest priority is assigned to PUs, the next priority is assigned to the interrupted SUs, and the last priority is assigned to the non-interrupted SUs. The second priority queue is simply a feedback queue that interrupted SUs return to that queue. However this model assumes a unitary class of SU and therefore does not take into account the different delay requirements of SUs. 
Multiple interruption in the middle of the transmission of a SU, will increase the data delivery time of the SU which leads to decrease of QoS for SUs therefore, spectrum usage behaviors in CRNs need to be analyzed to minimize SUs handoff delay. In order to minimize and characterize multiple handoff delay, M/G/1 queuing model of mixed PRP/NPRP is proposed in this paper. In the proposed queuing model the SUs are divided into two classes. SUs with delay sensitive services assigned higher priority class than SUs with delay insensitive services. Furthermore, the model assigns higher priority for the SUs that have been interrupted over uninterrupted SUs for each class of SU to decrease the handoff delay of the SUs that experience multiple interruptions.

\section{PROPOSED SYSTEM MODEL}

In the proposed model, the CRN is assumed to be a time-slotted system [11] which means each user's data is divided into time slots of equal sizes. Each time slot consists of two parts as shown in Figure 2. The first part of the time slot performs spectrum sensing while the other part is for transmitting data. In the first part, the SU periodically monitors the channel, when it senses that the current channel is not busy, then in the second part the transmission will commence while the procedure of spectrum handoff must be achieved if the SU senses that the channel is busy [9].

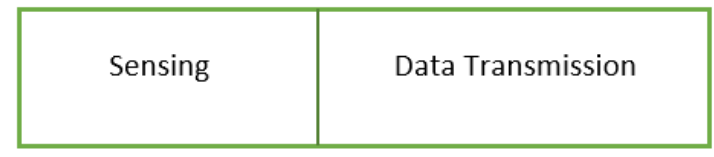

Figure 2. The structure of the time slots of the secondary networks

In the proposed system model, access points are suggested for uplink and downlink for PUs and SUs to compete for spectrum channels utilization. The suggested model comprises of two channels that are independent of each other. The channels are modelled using M/G/1 queuing theorem based on (PRP/NPRP) as illustrated in Figure 3. Each of the wireless channels consists of five priority queues. The queue of higher priority is dedicated for PUs, and the rest queues allocated to SUs. Two queues are for new arriving SUs of two classes, the first class of SU for delay sensitive services and the second class of SU is for delay insensitive services, and the two rest queues are for interrupted SUs for each class of SU. For simplicity, the queues are performed with infinite length.

In this model, PUs can interrupt the ongoing transmissions of SUs so that the interaction between them is PRP. It is assumed that SUs can't interrupt each other to avoid frequent spectrum handoff for SUs with lower priorities which means that the interaction between SUs is NPRP. Class 1 SU (SU1) has higher priority than class 2 SU (SU2) and interrupted SUs for each class has higher priority than the new arriving SU (uninterrupted SUs). For users of the same type in the same queue the sequence of the channel access is first come first served (FCFS). Throughout SU transmission it may encounter multiple interruptions from PUs. The unfinished transmission of SUs that have been interrupted can be resumed instead of retransmission of the whole connection.

Assuming the primary users PUs and both 1st and 2nd secondary users classes SU1 and SU2 reach at their target channel $\mathrm{k}$ in accordance with Poisson processes of mean rates $\lambda_{p}^{k}, \lambda_{s 1}^{k}$ and $\lambda_{s 2}^{k}$ respectively, and their mean service time that is generally distributed are $\mathrm{E}\left[X_{p}^{k}\right], \mathrm{E}\left[X_{s 1}^{k}\right]$ and $\mathrm{E}\left[X_{s 2}^{k}\right]$. When the users arrive at a busy channel they will have to wait in their queues till the channel becomes idle. The interrupted SUs are represented by SU with $i$ interruptions $(i \geq 1)$ and will reach at their desired channel with accordance to Poisson process too of mean rates $\lambda_{s 1, i}^{k}$ and $\lambda_{s 2, i}^{k}$ for 1 st and 2nd secondary users classes respectively and mean service time of $\mathrm{E}\left[X_{s 1, i}^{k}\right]$ and $\mathrm{E}\left[X_{s 2, i}^{k}\right]$. SUs parameters with zero interruptions (i=0) are denoted with $\lambda_{s 1}^{k}, \lambda_{s 2}^{k}, \mathrm{E}\left[X_{s 1}^{k}\right]$ and $\mathrm{E}\left[X_{s 2}^{k}\right]$.

When SU is interrupted and chose to stay at its channel, it'll be put at the head of the interrupted SU queue of its class but if it chose to change the channel, it will be put at the tail of the interrupted SU queue of its class. Figure 3 shows that the primary user is transmitting over channel K', while at channel K a SU of class 2 priority is transmitting when a PU reaches and interrupts the SU, this SU will either switch to channel $\mathrm{K}$ ' that is appeared by the 'yes' branch which comes after the 'switch' box in Figure 3, it will be placed at the tail of the interrupted SU2 queue (ISU2 queue). Or it will stay at the current channel that appeared by the 'NO' branch and it will be placed at the head of the interrupted SU2 queue (ISU2 queue). 


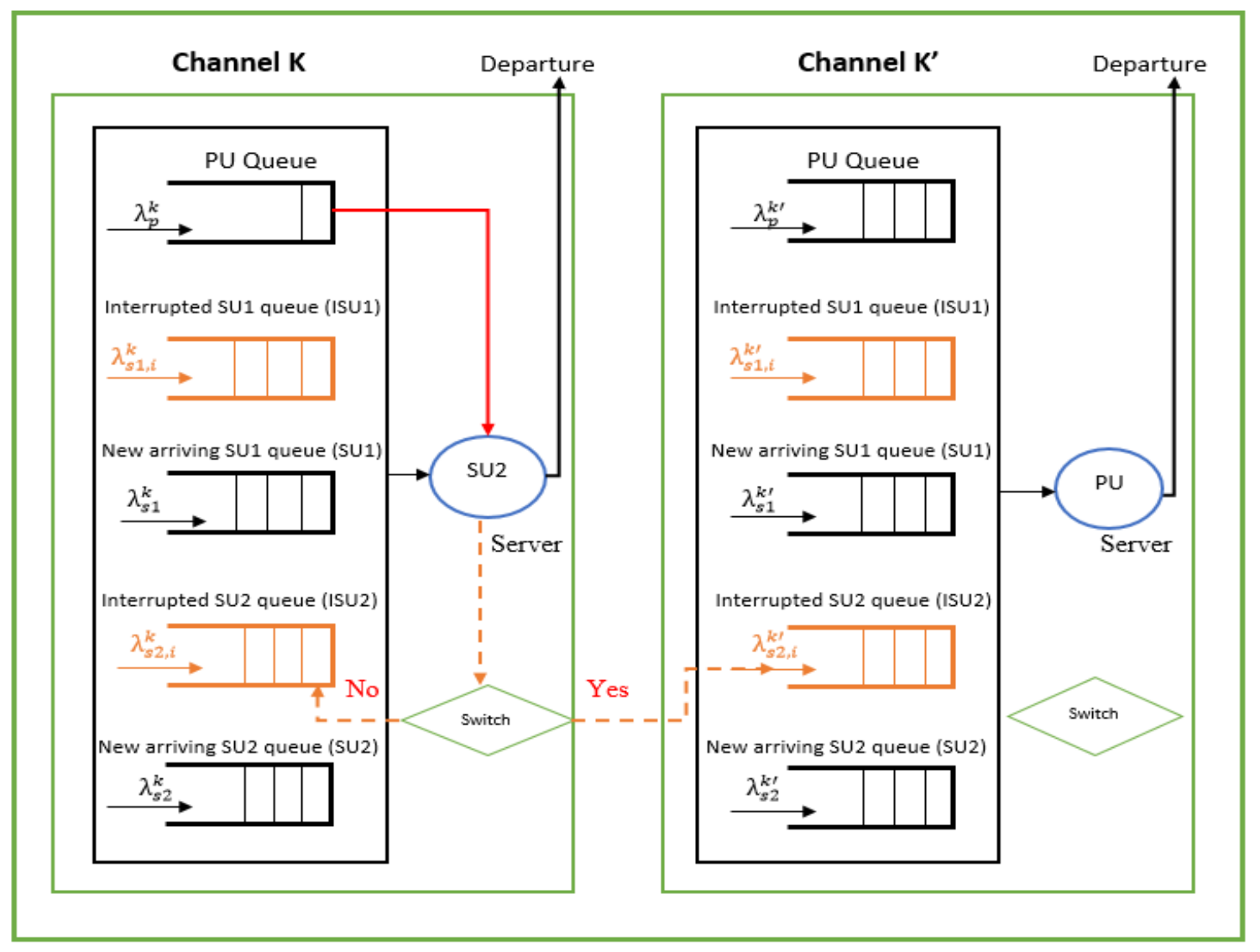

Figure 3. Mixed PRP/NPRP queueing model for spectrum handoff schemes

To ease the present analyses, the following assumptions are considered [11]:

a) The primary users are preassigned with a target channel or default channel. Also, spectrum decision algorithms select a target channel for each secondary user.

b) SUs can sense and detect the presence of PUs perfectly.

c) At each channel, only one user in a time can transmit its data.

\section{MODEL ANALYSIS}

In the present part, a mathematical framework for the handoff delay and average extended data delivery time for SUs is evaluated and analyzed that is considered as an essential measure of performance of SUs traffic. The utilization factors of PUs and SUs are respectively defined as [11,20]:

$$
\begin{aligned}
& p_{p}^{k}=\lambda_{p}^{k} \mathrm{E}\left[X_{p}^{k}\right] \\
& p_{s 1, \mathrm{i}}^{k}=\lambda_{s 1, \mathrm{i}}^{k} \mathrm{E}\left[X_{s 1, \mathrm{i}}^{k}\right] \\
& p_{s 2, \mathrm{i}}^{k}=\lambda_{s 2, \mathrm{i}}^{k} \mathrm{E}\left[X_{s 2, \mathrm{i}}^{k}\right]
\end{aligned}
$$

And the overall system utilization is described by:

$$
p^{k}=p_{p}^{k}+\sum_{i=0}^{n_{\max }} p_{s 1, i}^{k}+\sum_{i=0}^{n_{\max }} p_{s 2, i}^{k}
$$

where $n_{\max }$ is the maximum number of interruptions. For simplicity, it is assumed that all the channels have the same parameters and they are identical. So, the notation (k) can be dropped in all system parameters:

$$
p=p_{p}+\sum_{i=0}^{n_{\max }} p_{s 1, i}+\sum_{i=0}^{n_{\max }} p_{s 2, i}
$$


The systems stability's important and sufficient conditions are:

$$
p<1, p_{p}<1,\left(\sum_{i=0}^{n_{\max }} p_{s 1, i}\right)<1,\left(\sum_{i=0}^{n_{\max }} p_{s 2, i}\right)<1
$$

\subsection{Calculations of the handoff delay}

SU connection's handoff delay is the duration from when SUs transmission is interrupted till it is resumed again. Define E[D] as the handoff delay for SUs. Each time, an interruption occurs, the SU will have to decide to remain at the current channel or to switch to other channels so, the cumulative handoff delay will be based on selecting the desired channel sequence. The handoff delay in this thesis, will be derived based on the desired channel sequence identified in the standard of IEEE 802.22 wireless regional area network (WRAN) [21]; the switching case and the non-switching case

\subsubsection{Switching case (always change strategy):}

In this strategy, after each interruption the SU chooses to switch to another channel and wait in the tail of the interrupted queue of its priority of the new channel and will be resumed after all the queued high priority PUs, SUs of higher priority, interrupted SUs of its priority present in the queue ahead of him, new arriving PUs and new arriving SUs of higher priority arrived in the waiting time of this SU finish their transmissions. Let $\mathrm{E}\left[W^{\prime}\right]$ be the the average waiting time of SUs in the changing approach, then the handoff delay $\mathrm{E}[D]$ in this strategy will be the sum of the waiting time $\left(\mathrm{E}\left[W^{\prime}\right]\right)$ and the switching delay $\left(\mathrm{t}_{s}\right)$. The handoff delay for class 1 SU (SU1) and class 2 SU (SU2) can be expressed respectively as:

$$
\mathrm{E}\left[D_{s 1}\right]=\mathrm{E}\left[W_{s 1}^{\prime}\right]+\mathrm{t}_{s} \text { and } \mathrm{E}\left[D_{s 2}\right]=\mathrm{E}\left[W_{s 2}^{\prime}\right]+\mathrm{t}_{s}
$$

$\mathrm{E}\left[W^{\prime}{ }_{s 1}\right]$ is the average waiting time of class $1 \mathrm{SU}$ (SU1) and can be calculated based on M/G/1 queue analysis $[22,23]$ as :

$$
\mathrm{E}\left[W^{\prime}{ }_{s 1}\right]=\mathrm{E}\left[R_{s}\right]+\mathrm{E}\left[N_{p}\right] \mathrm{E}\left[X_{p}\right]+\sum_{i=1}^{n_{\max }} \mathrm{E}\left[N_{s 1, i}\right] \mathrm{E}\left[X_{s 1, i}\right]+\lambda_{p} E\left[W^{\prime}{ }_{s 1}\right] \mathrm{E}\left[X_{p}\right]
$$

$\mathrm{E}\left[R_{s}\right]+\mathrm{E}\left[N_{p}\right] \mathrm{E}\left[X_{p}\right]+\sum_{i=1}^{n_{\max }} \mathrm{E}\left[N_{s 1, i}\right] \mathrm{E}\left[X_{s 1, i}\right]$ is Queued PUs and interrupted SU1s ahead of him in ISU1 queue, $\lambda_{p} E\left[W^{\prime}{ }_{s 1}\right] \mathrm{E}\left[X_{p}\right]$ is new arriving PUs

The first term in (7) represents the mean residual service time of the user presently being served at the time that the interrupted SU reaches at the channel. The user that is served currently may be a PU or SU of any class as in the proposed model SUs cannot preempt each other. The second term and the third term are for the accumulative waiting time that is produced by the PUs from PUs queue and interrupted SU1s. The fourth term of the equation is for the accumulative waiting time resulted from the newly arrived PUs that is arriving in the period of $\mathrm{E}\left[W^{\prime}{ }_{s 1}\right]$. The mean residual service time $\mathrm{E}\left[R_{s}\right]$ can be expressed as $[22,24]$ :

$$
\mathrm{E}\left[R_{s}\right]=\frac{1}{2} \lambda_{p} \mathrm{E}\left[X_{p}^{2}\right]+\frac{1}{2} \sum_{i=0}^{n_{\max }} \lambda_{s 1, i} \mathrm{E}\left[X_{s 1, i}{ }^{2}\right]+\frac{1}{2} \sum_{i=0}^{n_{\max }} \lambda_{s 2, \mathrm{i}} \mathrm{E}\left[X_{s 2, i}{ }^{2}\right]
$$

The first term of (8) represents the residual service time for the PU and the second term and third terms represent the residual service time for SU1 and SU2 with $i$ interruptions respectively, where $\mathrm{E}\left[X_{p}{ }^{2}\right]$ is the second moment of the service time for PU, $\mathrm{E}\left[X_{s 1, i}{ }^{2}\right]$ and $\mathrm{E}\left[X_{s 2, i}{ }^{2}\right]$ are the second moment of mean service time of SU1 and SU2 respectively.

Now, one can derive the second term of (7). $\mathrm{E}\left[N_{p}\right]$ is the average number of PUs that are queued using little's law:

$$
\mathrm{E}\left[N_{p}\right]=\lambda_{p} \mathrm{E}\left[W_{p}\right]
$$

where $\mathrm{E}\left[W_{p}\right]$ is the average waiting time of the PU connections, which is is the period from the time that a PU connection arrives at the high-priority queue of its default channel until it gets the opportunity to transmit its data. Hence, it follows that:

$$
\mathrm{E}\left[W_{p}\right]=\mathrm{E}\left[R_{p}\right]+\mathrm{E}\left[N_{p}\right] \mathrm{E}\left[X_{p}\right]
$$


where $\mathrm{E}\left[R_{p}\right]$ is the average residual service time resulting from only the PU connections as PU s have preemptive priority and can interrupt the ongoing transmission of SUs. The second term of (10) is for the total workload of PU connections in the PU queue. $\mathrm{E}\left[R_{p}\right]$ can be expressed as:

$$
\mathrm{E}\left[R_{p}\right]=\frac{1}{2} \lambda_{p} \mathrm{E}\left[X_{p}^{2}\right]
$$

Substituting (9) and (11) into (10) then (10) becomes:

$$
\mathrm{E}\left[W_{p}\right]=\frac{\mathrm{E}\left[R_{p}\right]}{\left(1-\lambda_{p} \mathrm{E}\left[x_{p}\right]\right)}=\frac{\lambda_{p} \mathrm{E}\left[x_{p}{ }^{2}\right]}{2\left(1-p_{p}\right)}
$$

and

$$
\mathrm{E}\left[N_{p}\right]=\frac{\lambda_{p}^{2} \mathrm{E}\left[x_{p}{ }^{2}\right]}{2\left(1-p_{p}\right)}
$$

Next, according to little's law, one can obtain:

$$
\mathrm{E}\left[N_{s 1, i}\right]=\lambda_{s 1, \mathrm{i}} \mathrm{E}\left[W_{s 1}^{\prime}\right]
$$

Finally substituting (13) and (14) into (7) and it becomes:

$$
\mathrm{E}\left[W^{\prime}{ }_{S 1}\right]=\frac{\mathrm{E}\left[R_{s}\right]+\frac{\lambda p^{2} \mathrm{E}\left[X_{p}^{2}\right]}{2\left(1-p_{p}\right)} \mathrm{E}\left[X_{p}\right]}{1-p_{p}-\sum_{i=1}^{n_{m} \max } p_{S 1, i}}=
$$

For SU2, the average waiting time of SU2 can be calculated in the same way as of SU1 but it is more complex because SU1 has higher priority than SU2 and SU2 will not be able to use the channel until all of the PUs and SU1s finish their connections. It could be expressed as:

$$
\begin{array}{r}
\mathrm{E}\left[W^{\prime}{ }_{s 2}\right]=\mathrm{E}\left[R_{s}\right]+\mathrm{E}\left[N_{p}\right] \mathrm{E}\left[X_{p}\right]+\sum_{i=1}^{n_{\max }} \mathrm{E}\left[N_{s 1, i}\right] \mathrm{E}\left[X_{s 1, i}\right]+\mathrm{E}\left[N_{s 1}\right] \mathrm{E}\left[X_{s 1}\right]+\sum_{i=1}^{n_{\max }} \mathrm{E}\left[N_{s 2, i}\right] \mathrm{E}\left[X_{s 2, i}\right]+\lambda_{p} \\
\mathrm{E}\left[W_{s 2}^{\prime}\right] \mathrm{E}\left[X_{p}\right]+\sum_{i=0}^{n_{\max }} \lambda_{s 1, \mathrm{i}} \mathrm{E}\left[W_{s 2}^{\prime}\right] \mathrm{E}\left[X_{s 1, i}\right]
\end{array}
$$

The first and second terms of (16) are the same as those of (7) that have been explained. While the third, fourth and fifth terms represent the accumulative waiting time produced by interrupted SU1s, and uninterrupted SU1s that are queued and interrupted SU2s present ahead of him in the ISU2 queue respectively. The sixth and seventh terms are for the accumulative waiting time produced by the newly arriving PUs, interrupted and uninterrupted SU1s arriving in the period of $\mathrm{E}\left[W^{\prime}{ }_{s 2}\right]$. Substituting (13) and (14) into (16) and using little's theorem yields:

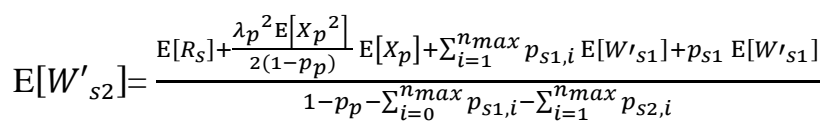

Next, substituting (8) and (15) in (17) produces:

$$
\mathrm{E}\left[W^{\prime}{ }_{s 2}\right]=\frac{\left(\mathrm{E}\left[R_{s}\right]+\frac{\lambda p^{2} \mathrm{E}\left[x_{p}^{2}\right]}{2\left(1-p_{p}\right)} \mathrm{E}\left[x_{p}\right]\right)\left(1-p_{p}+p_{s 1}\right)}{\left(1-p_{p}-\sum_{i=1}^{n_{\max }} p_{s 1, i}\right)\left(1-p_{p}-\sum_{i=0}^{n_{\max }} p_{s 1, i}-\sum_{i=1}^{n_{\max }} p_{s 2, i}\right)}
$$

Now, substituting (15) and (18) into (6) the handoff delay for SU1 and SU2 of the proposed system model for the always change strategy can be obtained:

$$
\mathrm{E}\left[D_{s 1}\right]=\frac{\mathrm{E}\left[R_{s}\right]+\frac{\lambda p^{2} \mathrm{E}\left[x_{p}^{2}\right]}{2\left(1-p_{p}\right)} \mathrm{E}\left[x_{p}\right]}{1-p_{p}-\sum_{i=1}^{n_{m} a x} p_{s 1, i}}+\mathrm{t}_{s}
$$

and 


$$
\mathrm{E}\left[D_{s 2}\right]=\frac{\left(\mathrm{E}\left[R_{s}\right]+\frac{\lambda_{p}{ }^{2}\left[x_{p}{ }^{2}\right]}{2\left(1-p_{p}\right)} \mathrm{E}\left[x_{p}\right]\right)\left(1-p_{p}+p_{s 1}\right)}{\left(1-p_{p}-\sum_{i=1}^{n \max } p_{s 1, i}\right)\left(1-p_{p}-\sum_{i=0}^{n_{\max }} p_{s 1, i}-\sum_{i=1}^{n_{\max }} p_{s 2, i}\right)}+\mathrm{t}_{s}
$$

\subsubsection{Non- switching case (always stay strategy)}

In this case, the SU connection after each interruption selects to stay at its default channel. In the non-switching case there is no switching (ts $=0$ ) so, the handoff delay is just the waiting time in a queue. If $E\left[W_{s 1}\right]$ is defined as the waiting time of SU1 and $E\left[W_{s 2}\right]$ as the waiting time of SU2 in the staying case then:

$$
E\left[D_{s 1}\right]=E\left[W_{s 1}\right], E\left[D_{s 2}\right]=E\left[W_{s 2}\right]
$$

Based on M/G/1 queue analysis, the waiting time for SU1 can be calculated as:

$$
E\left[W_{s 1}\right]=E\left[X_{p}\right]+\lambda_{p} \mathrm{E}\left[W_{s 1}\right] \mathrm{E}\left[X_{p}\right]
$$

The first term in (22) is for the mean service time of the primary user that is the reason of the interruption, and the second part of the equation shows the cumulative waiting time resulted from the new arrival of primary users arriving in the period of $\mathrm{E}\left[W_{s 1}\right]$. Solving (22) yields:

$$
E\left[W_{s 1}\right]=\frac{\mathrm{E}\left[x_{p}\right]}{\left(1-p_{p}\right)}
$$

And the interrupted SU of class 2 waiting time $\left[W_{s 2}\right]$ is obtained as:

$$
E\left[W_{s 2}\right]=\mathrm{E}\left[X_{p}\right]+\lambda_{p} \mathrm{E}\left[W_{s 2}\right] \mathrm{E}\left[X_{p}\right]+\sum_{i=0}^{n_{\max }} \lambda_{s 1, \mathrm{i}} E\left[W_{s 2}\right] \mathrm{E}\left[X_{s 1, i}\right]
$$

The first and second term of (24) are the same as those of (22), the third term represents the cumulative workload that has been resulted from new arrivals of the interrupted SU1 connections and uninterrupted SU1 connections arriving during $E\left[W_{s 2}\right]$. Solving (24) produces:

$$
\left.E\left[W_{s 2}\right]=\frac{E\left[X_{p}\right]}{\left(1-p_{p}-\sum_{i=0}^{n} \max \right.} p_{s 1, i}\right)
$$

Then, the handoff delays for SU1 and SU2 in the always stay case are:

$$
\begin{aligned}
& E\left[D_{s 1}\right]=\frac{\mathrm{E}\left[x_{p}\right]}{\left(1-p_{p}\right)} \\
& E\left[D_{s 2}\right]=\frac{E\left[x_{p}\right]}{\left(1-p_{p}-\sum_{i=0}^{n_{m} \max } p_{s 1, i}\right)}
\end{aligned}
$$

\subsection{The extended data delivery time}

The average extended data delivery time is the time duration between the beginning of transmitting data and finishing the whole transmission, through which multiple interruptions may happen. The average delivery time of the data of a SU who has experienced multiple interruptions throughout its transmission period can be calculated as [11]:

$$
E[T]=\sum_{i=1}^{n_{\max }} E[T \mid N=n] \operatorname{Pr}(N=n)
$$

where $\mathrm{N}$ is the total number of interruptions of this $\mathrm{SU}$.

The extended data delivery time for this SU includes the service time of the secondary user and the accumulative delay caused by multiple handoffs. The conditional mean of the extended data delivery time for the regarded $\mathrm{SU}$ given the event $\mathrm{N}=\mathrm{n}$ can be expressed as [11]:

$$
E[T \mid N=n]=\mathrm{E}\left[X_{S}\right]+\sum_{i=1}^{n_{\max }} E[\mathrm{D}]
$$

where $\mathrm{D}$ is the handoff delay of the regarded SU for the ith interruption. Let $p_{i}$ be the probability that a SU who have experienced interruptions is interrupted again. The probability of experiencing $\mathrm{n}$ times of interruption by this $\mathrm{SU}$ can be derived as: 


$$
\operatorname{Pr}(N=n)=\left(1-p_{n}\right) \prod_{i=0}^{n-1} p_{i}
$$

where $p_{i}=\lambda_{p} \mathrm{E}\left[X_{i}\right]$ from [11].

Finally substituting (29) and (30) in (28) yields:

$$
E[T]=\mathrm{E}\left[X_{s}\right]+\sum_{n=1}^{n_{\max }}\left[\left(\sum_{i=1}^{n} E[\mathrm{D}]\right)\left(1-p_{n}\right) \prod_{i=0}^{n-1} p_{i}\right]
$$

where $E[D]=\left\{\begin{array}{c}E\left[W_{s}\right] \text { for stay case } \\ E\left[W^{\prime}{ }_{s}\right]+t \text { f for the change case }\end{array}\right\}$

As shown in (31) can be rewritten for class one SU (SU1) for the stay case and change case respectively as:

$$
\begin{aligned}
& E\left[T_{s 1 \text { stay }}\right]=\mathrm{E}\left[X_{s 1}\right]+\sum_{n=1}^{n_{\max }}\left[\left(\sum_{i=1}^{n} E\left[W_{s 1}\right]\right)\left(1-p_{s 1, n}\right) \prod_{i=0}^{n-1} p_{s 1, i}\right] \\
& E\left[T_{s 1 \text { change }}\right]=\mathrm{E}\left[X_{s 1}\right]+\sum_{n=1}^{n_{\max }}\left[\left(\sum_{i=1}^{n} E\left[W_{s 1}^{\prime}\right]+t_{s}\right)\left(1-p_{s 1, n}\right) \prod_{i=0}^{n-1} p_{s 1, i}\right]
\end{aligned}
$$

Where $p_{s 1, i}=\lambda_{p} \mathrm{E}\left[X_{s 1, i}\right]$

Substituting (19) and (26) into (32) and (33) yields the extended data delivery time of SU1 for stay case and change case respectively. While for class 2 SU (SU2) the extended data delivery time for stay case and change case can be expressed respectively as:

$$
\begin{aligned}
& E\left[T_{s 2 \text { stay }}\right]=\mathrm{E}\left[X_{s 2}\right]+\sum_{n=1}^{n_{\max }}\left[\left(\sum_{i=1}^{n} E\left[W_{s 2}\right]\right)\left(1-p_{s 2, n}\right) \prod_{i=0}^{n-1} p_{s 2, i}\right] \\
& E\left[T_{s 2 \text { change }}\right]=\mathrm{E}\left[X_{s 2}\right]+\sum_{n=1}^{n_{\max }}\left[\left(\sum_{i=1}^{n} E\left[W_{s 2}^{\prime}\right]+t_{s}\right)\left(1-p_{s 2, n}\right) \prod_{i=0}^{n-1} p_{s 2, i}\right]
\end{aligned}
$$

where, $p_{s 2, i}=\lambda_{p} \mathrm{E}\left[X_{s 2, i}\right]$

Substituting (20) and (27) in (34) and (35) produces the extended data delivery time of SU2 for stay case and change case respectively.

Depending on the analytical results, SUs can adaptively decide which target channel sequence is better to minimize their extended data delivery time. Therefore, according to the principle of adaptive channel selection, the average delivery time of the data can be expressed as:

$$
\mathrm{E}[\mathrm{T}]=\min \left(E\left[T_{\text {stay }}\right], E\left[T_{\text {change }}\right]\right)
$$

\section{RESULTS AND DISCUSSION}

The suggested model's performance is evaluated by using the delay performance measures and compared it with the models introduced in [11] and [16]. In the experiments, the duration of time slot that is used is $10 \mathrm{msec}$ which is recommended by the standard of IEEE 802.22. It is assumed that PUs and SUs service time to be considered an exponential distribution with SUs service time of $\mathrm{E}\left[X_{\text {su1 }}\right]=$ $\mathrm{E}\left[X_{s u 2}\right]=8$ (slots/arrival) and PU service time of $\mathrm{E}\left[X_{p}\right]=25$ (slots/arrival), furthermore it is assumed that $\lambda_{s u 1}=\lambda_{s u 2}=0.0075$. A range for $\lambda_{p}$ as [0,0.0325] is selected in order to cover a high range of utilization and to satisfy the stability condition $p<1$. It is considered that the two channels have the same parameters and the channel switching delay $t s$ is neglected as it has a small value and we set $n_{\max }=5$.

In order to validate the proposed analytical model, a simulation environment is set up similar to the model described in Section 2, where the model parameters are chosen as given above. A MATLAB Simulink model has been developed using SimEvents toolbox that is used in modeling a discrete event system [25].

Figure 4 and 5 shows a comparison between analytical and simulation results for the stay case and change case respectively. From the graphs it is clear that the analytical and simulation results are approximately the same which validate the proposed model. In addition from the figures we can conclude that $\lambda_{p}$ and the average extended data delivery time for SUs $(E[T])$ increase linearly. As the arrival rate of the PUs grows, the extended data delivery time $(E[T])$ for SUs of both classes and for both stay case and change case grows also. As $\lambda_{p}$ increases, the chance of finding an idle channel for SU reduces, also the interruption probability grows and this increases the handoff delay and the extended data delivery time. 


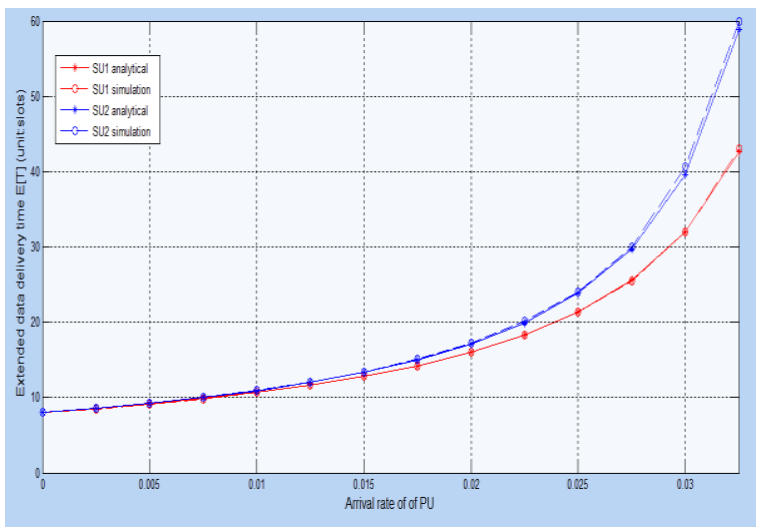

Figure 4. Comparison between the analytical results and simulation results for the stay case

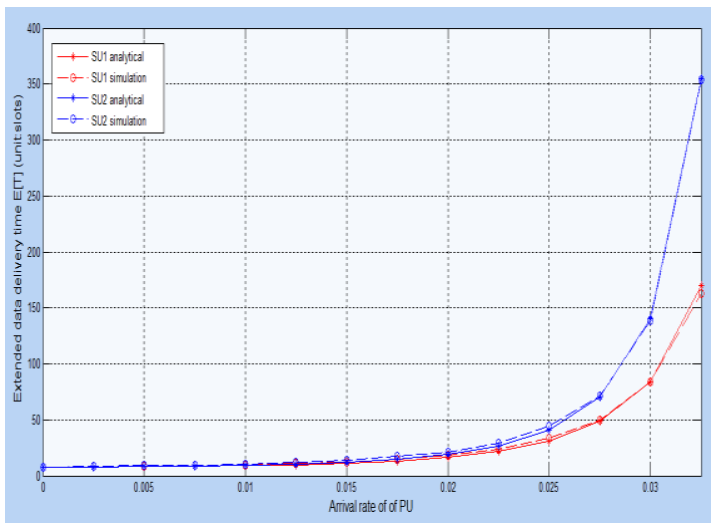

Figure 5. Comparison between the analytical results and the simulation results for the change case

Figure 6 and Figure 7 illustrates the comparison of the extended data delivery time for the nonswitching (always stay) and switching (always change) spectrum handoff strategies and the traffic adaptive target channel selection principle for SU1 and SU2 respectively. It is shown that for the switching case, higher values of $\lambda_{p}$ will raise the probability of having higher waiting time for SUs. The traffic adaptive channel selection, can appropriately select a better target channel according to traffic conditions. In Figure 6 it is shown that a cross point occurs when $\lambda_{p}$ is equal to 0.0195 arrivals /slot, where the always stay strategy and always change strategy results in the same extended data delivery time. The SU choses to switch to another channel for lower values of $\lambda_{p}\left(\lambda_{p}>0.0195\right)$, while for higher values of $\lambda_{p}\left(\lambda_{p}<0.0195\right)$ it decides to stay at its channel.

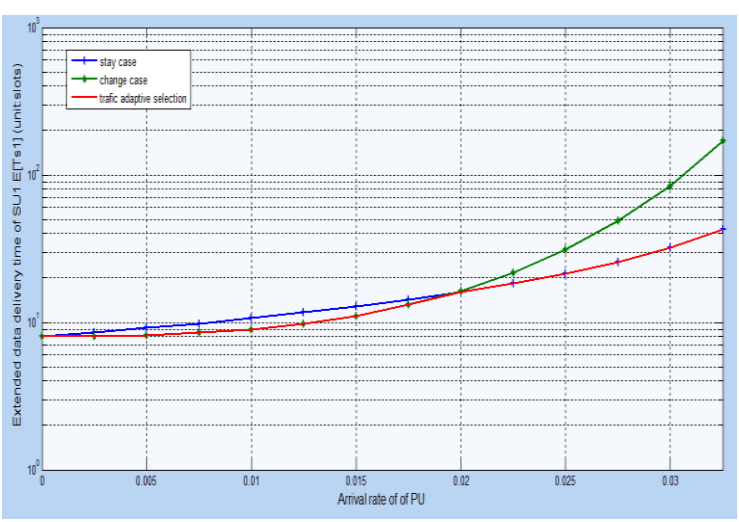

Figure 6. Comparison between the extended data delivery time of the stay case and change case of SU1 as well as the traffic adaptive target channel selection principle

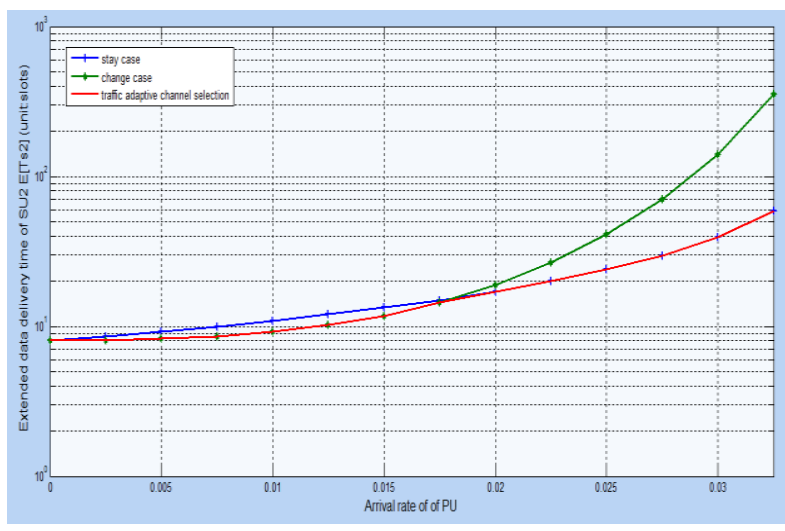

Figure 7. Comparison between the extended data delivery time of the stay case and change case of SU2 as well as the traffic adaptive target channel selection principle

Figure 8 illustrates the comparison between the suggested model in this thesis and the model presented in [16] for the change case. The performance of the proposed model is better in comparison with the model presented in [16] since it decreases the extended data delivery time, specifically for the high values of $\lambda_{p}$. For the stay case, the extended data delivery time of the proposed model is approximately the same as of the model in [16] when the interrupted SU choses to stay at its channel, it will be pushed in the head of the queue of its priority. This means, it has higher priority than all the waiting users so separating a queue for the interrupted users for the stay case doesn't make difference, while for the change case it improves the performance significantly. Figure 9 illustrates the comparison of the proposed model with the unitary SU model presented in [11] for the change case. The average extended data delivery time for the two classes of 
$\mathrm{SU}$ is calculated as $\left[T_{a v}\right]=\frac{\lambda_{s 1} E\left[T_{s 1} \text { change }\right]+\lambda_{S 2} E\left[T_{S 2 \text { change }}\right]}{\lambda_{S 1}+\lambda_{S 2}}=\frac{E\left[T_{s 1 \text { change }}\right]+E\left[T_{s 2 \text { change }}\right]}{2}$, It is clearly shown that the suggested model has a better performance when the interrupted user is assigned a higher priority to reduce the handoff delay and the extended data delivery time for the change case, while preserves it for the always stay case.

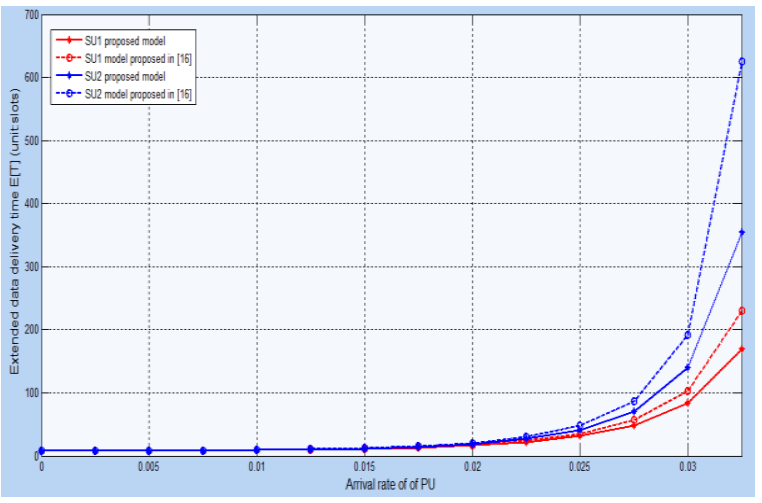

Figure 8. Comparison for the change case between the suggested model and the model proposed in [16].

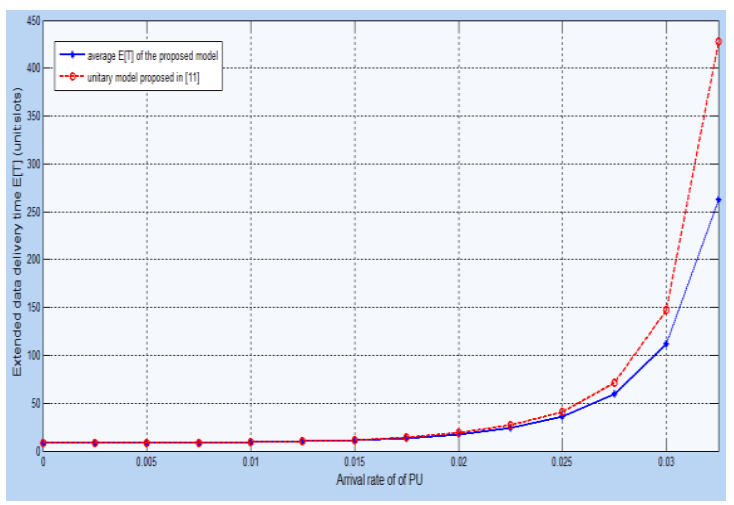

Figure 9. Comparison of the suggested model with the unitary model proposed in [11]

\section{CONCLUSION}

In the present paper, M/G/1 queuing model based on a mixed preemptive non-preemptive resume priority PRP/NPRP has been proposed to characterize multiple handoff delay. In the proposed queuing model the SUs traffic is classified into two priority classes the highest priority class is assigned for the delay sensitive services whereas the lowest priority class is assigned for delay insensitive services. Furthermore, the model assigns higher priority for the SUs that has experienced interruption over uninterrupted SUs (new arriving SUs) for both classes of SU for the purpose of decreasing the handoff delay for the secondary users that experienced interruption. The proposed model has analyzed and evaluated the average extended data delivery time for two different proactive spectrum handoff strategies (non-switching and switching strategies). Numerical results have indicated that the suggested model performs better than the existing models and decreases the extended data delivery time for both SU classes for the change case, while preserves it for the always stay case.

\section{REFERENCES}

[1] I. F. Akyildiz, W. Y. Lee, M. C. Vuran, and S. Mohanty, "NeXt generation/dynamic spectrum access/cognitive radio wireless networks: A survey," Comput. Networks, vol. 50, no. 13, pp. 2127-2159, 2006.

[2] C. Hernandez, D. Giral, and C. Salgado, "Failed handoffs in collaborative Wi-Fi networks," TELKOMNIKA (Telecommunication Comput. Electron. Control), vol. 18, no. 2, pp. 669-675, 2020.

[3] Y. C. Liang, K. C. Chen, G. Y. Li, and P. Mähönen, "Cognitive radio networking and communications: An overview," IEEE Trans. Veh. Technol., vol. 60, no. 7, pp. 3386-3407, 2011.

[4] C. Hernandez, J. Rodriguez, and D. Giral, "Spectrum allocation model for cognitive wireless networks based on the Artificial Bee Colony algorithm,” Indones. J. Electr. Eng. Comput. Sci., vol. 14, no. 2, pp. 257-266, 2019.

[5] M. Song, C. Xin, Y. Zhao, and X. Cheng, "Dynamic spectrum access: From cognitive radio to network radio," IEEE Wirel. Commun., vol. 19, no. 1, pp. 23-29, 2012.

[6] J. Mitola and G. Q. Maguire, "Cognitive radios: Making software radios more personal," IEEE Pers. Commun., vol. 6, no. 4, pp. 13-18, 1999.

[7] K. Kumar, A. Prakash, and R. Tripathi, "Spectrum handoff in cognitive radio networks: A classification and comprehensive survey," J. Netw. Comput. Appl., vol. 61, pp. 161-188, 2016.

[8] A. Shakeel, et al, "Adaptive Spectrum Handoff Scheme in Cognitive Radio Ad-Hoc Networks," 2018 International Conference on Advances in Computing and Communication Engineering (ICACCE), pp. 127-132, 2018.

[9] S. Zahed, I. Awan, and A. Cullen, "Analytical modeling for spectrum handoff decision in cognitive radio networks," Simul. Model. Pract. Theory, vol. 38, pp. 98-114, 2013.

[10] J. Thomas and P. P. Menon, "A survey on spectrum handoff in cognitive radio networks," 2017 International Conference on Innovations in Information, Embedded and Communication Systems (ICIIECS), pp. 1-4, 2018.

[11] L. C. Wang, C. W. Wang, and C. J. Chang, "Modeling and Analysis for Spectrum Handoffs in Cognitive Radio Networks," IEEE Trans. Mob. Comput., vol. 11, no. 9, pp. 1499-1513, 2012. 
[12] C.-W. Wang and L.-C. Wang, "Modeling and analysis for proactive-decision spectrum handoff in cognitive radio Networks," 2009 IEEE Int. Conf. Commun., pp. 1-6, 2009.

[13] S. T. Talat, C.-W. Wang, and L.-C. Wang, "Spectrum decision for cognitive radio networks with variousbandwidth channels," 2016 IEEE Wireless Communications and Networking Conference, pp. 1-6, 2016.

[14] I. Suliman and J. Lehtomäki, "Queueing analysis of opportunistic access in cognitive radios," 2009 2nd Int. Work. Cogn. Radio Adv. Spectr. Manag, pp. 153-157, 2009.

[15] Y. Wu, F. Hu, S. Kumar, M. Guo, and K. Bao, "Spectrum handoffs with mixed-priority queueing model over Cognitive Radio Networks,” 2013 IEEE Glob. Conf. Signal Inf. Process. Glob. 2013 - Proc., pp. 1194-1197, 2013.

[16] [Y. Wu, et al, "A learning-based QoE-driven spectrum handoff scheme for multimedia transmissions over cognitive radio networks," IEEE J. Sel. Areas Commun., vol. 32, no. 11, pp. 2134-2148, 2014.

[17] H. Tran, T. Q. Duong, and H. J. Zepernick, "Average waiting time of packets with different priorities in cognitive radio networks," IEEE 5th International Symposium on Wireless Pervasive Computing 2010, pp. 122-127, 2010.

[18] L. Zhang, T. Song, M. Wu, X. Bao, J. Guo, and J. Hu, "Traffic-adaptive proactive spectrum handoff strategy for graded secondary users in cognitive radio networks," Chinese J. Electron., vol. 24, no. 4, pp. 844-851, 2015.

[19] M. Fahimi and A. Ghasemi, "Analysis of the PRP M/G/1 queuing system for cognitive radio networks with handoff management," 2014 22nd Iranian Conference on Electrical Engineering (ICEE), pp. 1047-1051, 2014.

[20] S. B.-H. Ng Chee-Hock, Fundamentals with applications in communication networks, 2nd Edition, 2008.

[21] W. Caldwell, et al,"IEEE 802.22: The first cognitive radio wireless regional area network standard," IEEE Commun. Mag., vol. 47, no. 1, pp. 130-138, 2009.

[22] S. K. Bose, An Introduction to Queueing Systems, Boston, MA: Springer US, 2002.

[23] M. Haviv, "The M / G / 1 Queueing System," Oper. Res., pp. 1-6, 2014.

[24] I. A. and J. Resing, Queueing theory, 2015.

[25] The MathWorks, "Simevents Users Guide," The MathWorks, Inc. Natick, MA, 2015. 